\title{
Violencia política contra las mujeres en razón de género y feminicidio.
}

\author{
Gazca Herrera Luis Alejandro ${ }^{1}$, Lezama Martínez Maty ${ }^{2}$, Cervantes Martínez Josué ${ }^{3}$
}

${ }^{1}$ Doctor en Administración Pública por el Instituto de Administración Pública A. C. - México, Maestro en Ciencias Administrativas y Licenciado en Sistemas Computacionales Administrativos por la Universidad Veracruzana - México, Profesor de Tiempo Completo- México. Consejero Local INE en Veracruz Orcid https://orcid.org/0000-0001-7637-2909. lgazca@uv.mx

${ }^{2}$ Licenciada en Derecho Universidad Veracruzana - México, Maestra en Derecho Procesal Centro Mexicano de Estudios de Posgrado México, Consejera Local del INE en el estado de Veracruz.Xalapa. México. Mty.ledezma@ine.mx

${ }^{3}$ Licenciado en Derecho Universidad Veracruzana México, Estudios de Maestría en Derecho Electoral Universidad de Xalapa - México, Consejero Presidente de la Junta Local Ejecutiva INE en el estado de Veracruz.Xalapa.México.Josue.cervantes@ine.mx

\section{DOI https://doi.org/10.46589/rdiasf.vi35.363}

Recibido 12 de marzo 2021.

Aceptado 2 de junio 2021

Publicado 30 de junio de 2021

\section{Resumen}

Es una realidad la existencia de actos de rezago y exclusión histórica en las que las mujeres han sido objeto, actos que lastiman y debilitan severamente a la democracia e impide el libre ejercicio de sus derechos. Hablar de la erradicación de la violencia política en razón de género y feminicidio, demanda y requiere del compromiso pleno de las diversas autoridades en el ámbito de sus respectivas competencias, de las y los actores políticos y, por supuesto de la sociedad misma. Este artículo tiene como objetivo presentar información relevante sobre la violencia política contra las mujeres y feminicidio, esto con el fin de razonar la problemática que se presenta en México, identificando una falta de cultura de respeto a los derechos políticos y electorales de las mujeres. 
El presente documento se desarrolló basado en una investigación de tipo descriptiva, para lo cual se realizó la revisión conceptual y literaria pertinente considerando documentos nacionales e internacionales sobre los conceptos en cuestión. Como resultados de la investigación se encontró que, aunque se tienen avances significativos en cuanto a la tutela jurídica de los derechos políticos y electorales de las mujeres actualmente representa es una lucha inacabada.

Palabras clave: Feminicidios, Razón de Género, Política, Elecciones, Cargos de elección popular

\begin{abstract}
The existence of acts of historical backwardness and exclusion in which women have been subjected, acts that severely hurt and weaken democracy and impede the free exercise of their rights is a reality. Talking about the eradication of political violence based on gender and feminicide, demands and requires the full commitment of the various authorities in the field of their respective competencies, of the political actors and, of course, of society itself. This article aims to present relevant information on political violence against women and femicide, this in order to explain the problems that arise in Mexico, identifying a lack of culture of respect for women's political and electoral rights. This document was developed based on a descriptive research, for which the pertinent conceptual and literary review was carried out considering national and international documents on the concepts in question. As results of the investigation, it was found that, although there are significant advances in terms of the legal protection of the political and electoral rights of women, it currently represents an unfinished struggle.
\end{abstract}

Keywords: Femicides, Gender Reason, Politics, Elections, Popular election offices

\title{
Introducción
}

La lucha por el reconocimiento de los derechos político-electorales de la mujer ha sido una labor histórica en la búsqueda de la erradicación de brechas estructurales, sociales y culturales, así como de cualquier conducta o práctica que tenga como objetivo el menoscabo de sus derechos. En los últimos años, el avance en la participación política de la mujer ha sido exponencial, aunque es indudable que persisten obstáculos para su acceso a cargos públicos.

En México, Rosa Torres González fue la primera mujer en ocupar un cargo de elección popular al convertirse en regidora del ayuntamiento de Mérida en 1923; más adelante, a través de 
la modificación del artículo 115 constitucional, en 1947, se logró reconocer el derecho a las mujeres a votar y ser votadas en los procesos electorales municipales; sin embargo, es hasta el año de 1953 cuando se culmina con el reconocimiento constitucional de votar y ser votadas.

El avance paulatino en la implementación de cuotas en la norma electoral encuentra su auge con la reforma constitucional en materia político-electoral 2014, ya que significó un parteaguas en la participación en ese ámbito de las mujeres, finalmente, los partidos políticos debían postular el $50 \%$ de sus candidaturas encabezadas por mujeres, garantizando así, mayores probabilidades de lograr espacios públicos que las representen.

Es así como, derivado de la aplicación de diversas acciones afirmativas, así como de la progresiva emisión de disposiciones legales encaminadas a lograr una igualdad sustantiva, en México, el último Proceso Electoral Federal 2017-2018, tuvo como resultado que la Cámara de Diputadas y Diputados esté conformada por 241 mujeres y 259 hombres, lo que determina una diferencia de 18 curules. Por lo que respecta a la conformación del Senado de la República, esta refleja la integración de 63 mujeres y 65 hombres, lo que representa una diferencia de 2 escaños (INE 2018).

A nivel mundial, de acuerdo con ONU Mujeres, al primero de enero de 2020, Rwanda es el país con mayor número de mujeres en su parlamento, en la cámara baja existe una presencia del $61.3 \%$, mientras que, en la cámara alta o senado, el 38.5\%. México ocupa el quinto lugar al situarse con el $48.2 \%$ y $49.2 \%$ respectivamente (ONU Mujeres 2020).

Sin embargo, la participación política de la mujer en la postulación y ocupación de cargos unipersonales es una tarea pendiente, ya que, a partir de su reconocimiento al derecho al voto y ser votadas, aún no se logra una participación importante en dichos cargos; en México, la Presidencia de la República no es la excepción, toda vez que, únicamente 6 mujeres se han disputado el cargo sin tener éxito alguno.

Es una realidad la permanencia de obstáculos que han enfrentado las mujeres para figurar de manera protagónica en la vida pública y política en el país; las conductas inhibitorias derivadas por la cultura patriarcal predominante, el rezago histórico y las herramientas legislativas poco efectivas respecto al blindaje en su participación han retrasado los índices positivos de su intervención en el terreno público.

Como se expresó anteriormente, en los últimos años, la participación política de la mujer ha incrementado significativamente, desafortunadamente, se estima que el incremento de su participación en los cargos de elección popular es acompañado también por un crecimiento en las prácticas que pudieran constituir el menoscabo, violación a sus derechos y en casos extremos, constituirse en feminicidios. 


\section{Marco Referencial}

El rezago tradicional que han sufrido las mujeres de manera histórica obliga al enfrentamiento constante de obstáculos en el ejercicio de sus derechos; por lo que respecta a su participación pública y política, se focaliza en el ejercicio de estos para ser candidatas, electas y finalmente para ejercer el poder político efectivamente.

La Ley General de Acceso de las Mujeres a una Vida Libre de Violencia, determina que la violencia política contra las mujeres en razón de género es toda acción u omisión, incluida la tolerancia, basada en elementos de género y ejercida dentro de la esfera pública o privada, que tenga por objeto o resultado limitar, anular o menoscabar el ejercicio efectivo de los derechos políticos y electorales de una o varias mujeres, el acceso al pleno ejercicio de las atribuciones inherentes a su cargo, labor o actividad, el libre desarrollo de la función pública, la toma de decisiones, la libertad de organización, así como el acceso y ejercicio a las prerrogativas, tratándose de precandidaturas, candidaturas, funciones o cargos públicos del mismo tipo. Se entenderá que las acciones $\mathrm{u}$ omisiones se basan en elementos de género, cuando se dirijan a una mujer por su condición de mujer; le afecten desproporcionadamente o tengan un impacto diferenciado en ella (CNPEVCM 2016).

Cabe precisar que la Ley General en Materia de Delitos Electorales (LGMDE) no tipificaba a la violencia política contra las mujeres en razón de género, siendo que en el último Proceso Electoral Federal 2017-2018, la Fiscalía Especializada para la Atención de Delitos Electorales, abrió 6 carpetas de investigación por Violencia Política de Género y 41 números de atención a través de la identificación de 8 conductas delictivas relacionadas con dicha Violencia y contenidas en la mencionada ley (FEPADE 2018).

De ahí que resulte relevante la expedición, el 13 de abril de 2020, del Decreto por el que se reforman y adicionan diversas disposiciones de la Ley General de Acceso a las Mujeres a una Vida Libre de Violencia, de la Ley General de Instituciones y Procedimientos Electorales, de la Ley General del Sistema de Medios de Impugnación en Materia Electoral, de la Ley General de Partidos Políticos, de la Ley General en Materia de Delitos Electorales, de la Ley Orgánica de la Fiscalía General de la República, de la Ley Orgánica del Poder Judicial de la Federación y de la Ley General de Responsabilidades Administrativas.

Ahora bien, de acuerdo con ONU Mujeres (2017), la violencia contra las mujeres y las niñas es una de las violaciones a derechos humanos más graves, extendida y arraigada en el mundo. Esta violencia es tanto causa como consecuencia de la desigualdad y de la discriminación de género.

Aunado a lo anterior, la Comisión Nacional para Prevenir y Erradicar la Violencia contra las Mujeres CNPEVCM (2016) califica al feminicidio como la forma más extrema de violencia contra la mujer, que tiene su origen en la desigualdad de género, es decir, en la posición de 
subordinación, marginalidad y riesgo en el cual éstas se encuentran respecto de los hombres (. Por su parte, la LGAMVLV señala que se entenderá por Violencia Feminicida a "la forma extrema de violencia de género contra las mujeres, producto de la violación de sus derechos humanos, en los ámbitos público y privado, conformada por el conjunto de conductas misóginas que pueden conllevar impunidad social y del Estado y puede culminar en homicidio y otras formas de muerte violenta de mujeres".

De acuerdo con información reportada por el Observatorio de Igualdad de Género de América Latina y el Caribe de la Comisión Económica Para América Latina y el Caribe, durante el año 2019, se registraron un total de 4,640 mujeres víctimas de feminicidio. Además, señala que los países con mayor incidencia son Brasil con 1941, seguido de México con 983 y Argentina con 252 registros (CEPAL 2019).

En México, el feminicidio es un problema serio, aun con la implementación de políticas públicas, recomendaciones internacionales, así como de la adopción de medidas de seguridad encaminadas a erradicar los feminicidios, de acuerdo con la información sobre violencia contra las mujeres emitida por el Secretariado Ejecutivo del Sistema Nacional de Seguridad Pública, en el país, se observa un incremento en los presuntos delitos de feminicidio, ya que para el año 2015, se registraron 411, mientras que, al mes de octubre 2020, la cifra aumentó a 777 . Además, señala que las entidades con mayor registro de presuntos delitos de feminicidio del periodo comprendido de enero a octubre de 2020, es el Estado de México (119), seguido de Veracruz (71) y Ciudad de México (61). De la misma manera, durante la periodicidad mencionada, los municipios con mayor número de presuntos feminicidios registrados son: Juárez (19), Tijuana (17) y Monterrey (15) (SESNSP 2020).

\section{Elecciones 2018 y feminicidio.}

El proceso electoral 2017-2018, tuvo como objetivo la celebración de la elección denominada como "la más grande de la historia", la simultaneidad en la renovación de 18,299 cargos de elección popular, tanto federales como locales, trajo consigo innumerables retos para las autoridades electorales, así como para la sociedad misma; sin embargo, también significó un sin número de oportunidades para el ejercicio pleno de los derechos político-electorales de las mujeres en la arena pública, para contender por la Presidencia de la República, en la ocupación de las curules y escaños del Congreso de la Unión, así como de aquellos cargos de índole estatal. Por lo que respecta a la contienda por la Presidencia de la República, únicamente se logró la participación de una candidata quien desistió de su postulación antes de la celebración de los comicios del 01 de julio 2018 (INE numeralia 2018).

Como se ha expresado anteriormente, la violencia política contra las mujeres en razón de género es una realidad innegable en el ejercicio de cargos públicos y/o en la postulación de los mismos, y comprende, entre otras, violencia física, psicológica, simbólica, sexual, patrimonial, económica y feminicida (CNDH 2018). 
Desafortunadamente, en México, los índices de violencia contra las mujeres políticas y candidatas son desalentadores, de acuerdo con el primer informe de violencia política contra las mujeres en México 2018, realizado por la Unidad de Análisis en Riesgos de Seguridad Etellekt Consultores, durante el Proceso Electoral 2017-2018, se registraron 382 agresiones en contra de políticos y candidatos en el país, de las cuales 93 han sido en contra de políticas y candidatas, 79 fueron agresiones directas con un saldo de 15 políticas asesinadas, de las cuales 4 obtuvieron la calidad de candidatas y 2 más precandidatas (Etellekt 2018).

De la misma manera, dicho informe señala que las entidades con mayores agresiones globales contra las mujeres políticas del periodo comprendido entre el 08 de abril y el 08 de mayo de 2018, son Guerrero, seguido de Puebla y Oaxaca. Además, precisa que, de las 93 políticas y candidatas agredidas, el 58\% pertenecían al ámbito municipal, $30 \%$ a nivel estatal y $12 \%$ a nivel federal. Ahora bien, se estima que la periodicidad durante la cual suceden con mayor frecuencia las agresiones dirigidas a las mujeres políticas es durante el periodo de campañas. Entre el 30 de marzo y el 02 de junio de 2018, se registraron un total de 66 agresiones, de las cuales 55 se dirigieron en contra de candidatas a puestos de elección popular. Desde el inicio de las campañas, ocurrieron 34 amenazas y 8 asesinatos en contra de políticas y candidatas en el país. Las entidades en las cuales ocurrieron los asesinatos sufridos por candidatas fueron: Guerrero (2), Oaxaca (1), Michoacán (1), Puebla (1) y Chihuahua (1) (Etellek 2018).

Otro dato para destacar es que las entidades federativas de Puebla y Ciudad de México encabezan amenazas e intimidaciones contra políticas. Además, del 08 de septiembre de 2017 al 02 de junio 2018, 44 mujeres políticas recibieron amenazas y actos de intimidación, de las cuales 34 eran candidatas, 5 precandidatas, 3 alcaldesas, 1 síndica y 1 diputada federal (Etellek 2018).

También, refleja que 67 candidatas y precandidatas fueron agredidas durante el mencionado proceso electoral, 39 constituyeron actos de intimidación y amenazas, 9 atentados contra familiares, 6 asesinatos, 5 agresiones físicas, 4 asaltos con violencia, 3 secuestros y 1 agresión con arma de fuego (Etellek 2018).

\section{Conclusiones}

En resumen, si bien hoy en día se tienen avances significativos en cuanto a la tutela jurídica de los derechos políticos y electorales de las mujeres, particularmente para garantizar, además de su participación en la postulación a los cargos públicos electos, el asegurar su efectividad forzando con medidas compensatorias el acceso a los mismos, la batalla por el reconocimiento político, social y administrativo es una lucha inacabada.

Las agresiones de carácter verbal, psicológico, moral, emocional y físico, llevadas al extremo del asesinato, tanto a candidatas como a sus familiares y personas cercanas, así como los obstáculos interpuestos para el libre ejercicio del cargo como la reducción de emolumentos o el omitir proporcionar información o elementos de trabajo, entre otros, fundamentalmente ejecutados por parte 
de hombres, revelan la resistencia subsistente a aceptar de manera libre y espontánea el reconocimiento de tales derechos.

Una asignatura pendiente en el campo jurídico era precisamente la de legislar en materia de violencia política en razón de género, rezago que llega a su fin el 13 de abril de 2020 en la esfera federal con la expedición del Decreto por el que se reforman y adicionan diversas disposiciones a ocho leyes sustantivas sobre el particular. Lo que sigue es garantizar su observancia, iniciando por su prevención.

\section{Referencias}

CEPAL. (2019). Feminicidio. Naciones Unidas CEPAL. Recuperado 20 de diciembre de 2020, de https://oig.cepal.org/es/indicadores/feminicidio

CNDH (2018). Violencia Política contra las Mujeres en Razón de Género. Pág. 2. Disponible en: https://www.cndh.org.mx/sites/all/doc/OtrosDocumentos/Doc 2018 056.pdf

CNPEVCM (2016).Comisión Nacional para Prevenir y Erradicar la Violencia Contra las Mujeres. ¿Qué es el feminicidio y cómo identificarlo? Gobierno de México. https://www.gob.mx/conavim/articulos/que-es-el-feminicidio-y-comoidentificarlo?idiom $=$ es $\#: \sim$ :text $=\mathrm{La} \% 20$ muerte $\% 20$ violenta $\% 20 \mathrm{de} \% 20 \mathrm{las}$, de $\% 20$ violencia $\% 20$ contra $\% 201 \mathrm{a} \% 20$ mujer.

Etellekt (2018). Consultores (2018, 14 junio). Primer Informe de Violencia Política contra Mujeres en México 2018. Etellekt. https://www.etellekt.com/reporte/primer-informe-de-violencia-politicamujeres.html

FEPADE (2018), Informe anual de actividades 2018, pág. 96 http://pgrstastdgfepade020.blob.core.windows.net/fepade/informes/Informefinal2018.pdf

INE (2018). Integración de la Legislatura Federal Elecciones 2018. Noviembre 25, 2020, de INE Sitio web: https://igualdad.ine.mx/elecciones/eleccion-2018/integracion-de-la-legislatura-federal/

INE numeralia (2018), Numeralia final proceso electoral 2017-2018. Disponible en: https://www.ine.mx/wp-content/uploads/2018/08/1Numeralia01072018-SIJE08072018findocx3.pdf

Instituto Nacional Electoral. (2018, 27 agosto). Integración de la Legislatura Federal. https://igualdad.ine.mx/elecciones/eleccion-2018/integracion-de-la-legislatura-federal/

ONU Mujeres. (2020). Mujeres en la política: 2020. Noviembre 28, 2020, de Organización de Naciones Unidas Sitio web: https://www.unwomen.org//media/headquarters/attachments/sections/library/publications/2020/women-in-politics-map2020-es.pdf?la $=$ es\&vs $=828$ 
ONU Mujeres. (2017). Violencia Feminicida en México. Diciembre 2, 2020, de Organización de Naciones Unidas Sitio web: https://www2.unwomen.org//media/field $\% 20$ office $\% 20$ mexico/documentos/publicaciones $/ 2019 /$ infografa $\% 20$ violencia $\% 20$ on u $\% 20$ mujeres $\% 20$ espaol_web.pdf?la $=$ es\&vs $=5828$

SESNSP. (2020). Información sobre violencia contra las Mujeres. Noviembre 29, 2020, de Secretariado Ejecutivo del Sistema Nacional de Seguridad Pública Sitio web: https://drive.google.com/file/d/1err78aRUv63aU1_HipYKE_VgAcEdC2tN/view

https://revistainvestigacionacademicasinfrontera.unison.mx/index.php/RDIASF/article/view/363

- Dia net

\section{iciteFatar \\ $\rightarrow$}
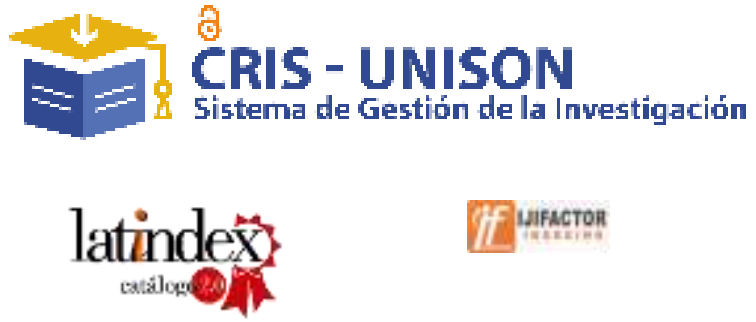

LatinREV Neliti - Indonesia's Research Repository

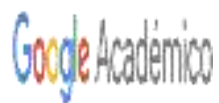

DORA

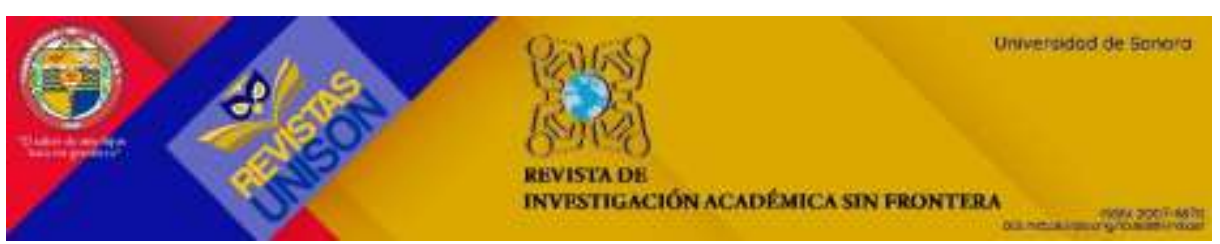

